\title{
Mucosite em pacientes portadores de câncer de cabeça e pescoço submetidos à radioquimioterapia*
}

\author{
MUCOSITIS IN HEAD AND NECK CANCER PATIENTS UNDERGOING \\ RADIOCHEMOTHERAPY
}

\author{
MUCOSITIS EN PACIENTES PORTADORES DE CÁNCER DE CABEZA Y CUELLO \\ SOMETIDOS A RADIOQUIMIOTERAPIA
}
Renata Cristina Schmidt Santos ${ }^{1}$, Rodrigo Souza Dias ${ }^{2}$, Adelmo José Giordani ${ }^{3}$, Roberto Araújo Segreto ${ }^{4}$, Helena Regina Comodo Segreto ${ }^{5}$

\section{RESUMO}

O objetivo do presente trabalho é classificar o grau de mucosite oral de acordo com os parâmetros internacionais do Common Toxicity Criterion (CTC) em pacientes portadores de tumor de cabeça e pescoço submetidos à radioterapia e quimioterapia concomitantes, e caracterizar um perfil dos pacientes em nosso meio, verificando os hábitos dos indivíduos, as características do tumor, o protocolo de tratamento e a intensidade desta reação aguda. Neste estudo foram avaliados 50 pacientes, submetidos à radioterapia em megavoltagem com doses entre 66 a 70 Gy e quimioterapia com cisplatina ou carboplatina concomitante. Semanalmente foi avaliado o grau de mucosite de acordo com o CTC, uma escala ordinal que apresenta 4 graus. Observou-se interrupção do tratamento por mucosite em $36 \%$ do total de pacientes e em $100 \%$ dos pacientes diabéticos, o que nos permitiu verificar que esta patologia contribui para a gravidade da mucosite.

\section{DESCRITORES}

Neoplasias de cabeça e pescoço

Radioterapia

Quimioterapia

Mucosite

Enfermagem oncológica

\begin{abstract}
The objective of present study was to classify oral mucositis according to the Common Toxicity Criterion (CTC) international parameters in head and neck tumor patients simultaneously treated with radio and chemotherapy, and characterize a patient profile in our area, observing the individuals' habits, tumor characteristics, treatment protocol and acute reaction intensity. Fifty patients undergoing simultaneous 66 to 70 Gy megavoltage radiotherapy and cisplatin/ carboplatin chemotherapy were evaluated in this study. Weekly evaluations of the degree of mucositis were perfoemed according to CTC, a four-degree ordinal scale; $36 \%$ of all patients and $100 \%$ of those with diabetes discontinued treatment due to mucositis, showing that this pathology contributes to the severity of mucositis.
\end{abstract}

\author{
DESCRIPTORS \\ Head and neck neoplasms \\ Radiotherapy \\ Drug therapy \\ Mucositis \\ Oncologic nursing
}

\begin{abstract}
RESUMEN
El trabajo objetivó clasificar el grado de Mucositis oral de acuerdo a parámetros internacionales del CTC en pacientes portadores de tumores de cabeza y cuello sometidos a radioterapia y quimioterapia concomitantes, y caracterizar un perfil de pacientes en nuestro medio, verificando hábitos de los individuos, características del tumor, protocolo de tratamiento e intensidad de esta reacción aguda. Fueron evaluados 50 pacientes sometidos a radioterapia en megavoltaje con dosis entre 66 y $70 \mathrm{G}$ y quimioterapia con cisplatino o carboplatino concomitante. Se evaluó semanalmente el grado de Mucositis según el Common Toxicity Criterio - CTC, una escala ordinal que presenta cuatro grados. Se observó interrupción del tratamiento por Mucositis en $36 \%$ del total de pacientes y en $100 \%$ de los pacientes diabéticos, lo que nos permite verificar que dicha patología potencia la gravedad de la mucositis.
\end{abstract}

\section{DESCRIPTORES}

Neoplasia de cabeza y cuello

Radioterapia

Quimioterapia

Mucositis

Enfermería oncológica

\footnotetext{
* Extraído da dissertação "Mucosite em pacientes portadores de câncer de cabeça e pescoço submetidos à radioterapia e quimioterapia concomitantes", Universidade Federal de São Paulo, 2009. ${ }^{1}$ Enfermeira. Mestre em Ciências pela Universidade Federal de São Paulo. São Paulo, SP, Brasil. re antena@yahoo.com.br ${ }^{2}$ Médico Assistente. Doutorando do Programa de Pós-Graduação em Radioterapia do Departamento de Oncologia da Universidade Féderal de São Paulo. São Paulo, SP, Brasil. rsdias@uninet.com.br ${ }^{3}$ Físico. Médico Coordenador do Programa de Especialização em Física-Médica do Setor de Radioterapia do Departamento de Oncologia da Universidade Federal de São Paulo. São Paulo, SP, Brasil. adelmogiordani@ig.com.br ${ }^{4}$ Professor Associado e Chefe do Setor de Radioterapia do Departamento de Oncologia da Universidade Federal de São Paulo. São Paulo, SP, Brasil. segreto.dmed@epm.br ${ }^{5}$ Professora Associada e Coordenadora do Laboratório de Radioterapia Experimental do Departamento de Oncologia da Universidade Federal de São Paulo. São Paulo, SP, Brasil. hrcs.dmed@epm.br
} 


\section{INTRODUÇÃO}

O câncer de cabeça e pescoço compreende todos os carcinomas originários do epitélio mucoescamoso, desde o lábio, cavidades oral e nasal, faringe, até a laringe e ouvido médio. É o terceiro tumor mais prevalente mundialmente e representa $7 \%$ dos 22,4 milhões de indivíduos com o diagnóstico de câncer, excluindo o câncer de pele não melanoma ${ }^{(1)}$.

Em relação ao Brasil, no ano de 2010, ocorreram cerca de 14.120 casos novos de câncer de boca. Este dado corresponde ao quinto câncer, com maior incidência no sexo masculino e o sétimo no sexo feminino(2).

Os tratamentos utilizados para esta enfermidade envolvem três modalidades: cirurgia, radioterapia e quimioterapia, que podem ser administrados de forma exclusiva ou concomitante ${ }^{(3)}$.

Os pacientes portadores de câncer de cabeça e pescoço que recebem radioterapia podem desenvolver reações na mucosa de diferentes intensidades. A associação de rádio e quimioterapia aumenta a incidência, severidade e duração da mucosite oral, especialmente quando são utilizadas combinações de diferentes drogas e esquemas de hiperfracionamento(4).

A mucosite causa dor significativa, dificuldade para mastigar e para deglutir e é considerada a reação aguda mais debilitante que surge durante o tratamento do câncer de cabeça e pescoço(3).

O mecanismo pelo qual ocorre a mucosite se baseia no fato de que a mucosa oral apresenta alta atividade mitótica e alto turnover celular. Devido ao alto grau de descamação celular, há necessidade contínua de multiplicação celular para recobrir a mucosa oral. Tecidos com alta atividade mitótica respondem rapidamente à radiação, uma vez que as fases mais sensíveis do ciclo celular são G2 e mitose. Desta forma a mucosa é rapidamente afetada ${ }^{(5)}$. 0 mesmo ocorre com os quimioterápicos, que são drogas que interferem no processo de proliferação e divisão celular. O fato das membranas mucosas sofrerem constante renovação torna-as extremamente sensíveis à ação destes quimioterápicos ${ }^{(5-6)}$.

Entre os métodos utilizados para avaliar o grau de mucosite na prática clínica, é importante ressaltar que a adequada avaliação da mucosa se faz necessária antes de iniciar a radioterapia e a quimioterapia em cabeça e pescoço, bem como durante o tratamento. Um bom método de avaliação deve considerar o relato do paciente, seu estado físico e nutricional e uma detalhada inspeção da cavidade oral ${ }^{(5,7)}$.

Entre as diversas escalas desenvolvidas para classificação da mucosite, análise de 400 trabalhos mostrou que
43\% dos estudos utilizam a escala do National Cancer Common Toxicity Criteria (NCl-CTC), 38\% utilizam World Health Organization (WHO), 10\% dos estudos empregam escalas específicas e $5 \%$ usam escalas de grupos colaboradores, tal como a escala usada pelo Radiation Therapy Oncology Group (RTOG) e a Eastern Cooperative Oncology Group (ECOG) $)^{(5)}$.

Neste estudo, utilizamos a escala do National Cancer Common Toxicity Criteria (NCl-CTC), pois esta possui uma versão em português que foi autorizada pelo Cancer Therapy Evaluation Program (CTEP), órgão do NCl responsável pela publicação da tabela(8).

Em vista da importância da mucosite durante a radioterapia e quimioterapia para tumores de cabeça e pescoço, a proposta do presente trabalho é classificar o grau de mucosite de acordo com os parâmetros internacionais do CTC 2.0 em pacientes submetidos à radioterapia e quimioterapia concomitantes e verificar se as características individuais dos pacientes, da doença e do tratamento têm influência sobre a incidência e a gravidade da mucosite.
A mucosite causa dor significativa, dificuldade para mastigar e para deglutir e é considerada a reação aguda mais debilitante tratamento do câncer de cabeça e pescoço

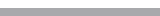

\section{REVISÃO DA LITERATURA}

Clinicamente observa-se a instalação da mucosite após a 20 semana do início da radioterapia. Inicia-se como processo inflamatório da mucosa, predisposição a infecções oportunistas e, dependendo da intensidade, pode evoluir para ulceração. Os sintomas são dor, dificuldade para comer, beber e falar. Como consequência, ocorre perda de peso e piora na condição geral do paciente ${ }^{(9)}$.

A mucosite inicialmente é caracterizada por morte das células epiteliais e ausência de substituição por novas células. Os vasos sanguíneos capilares se tornam hiperpermeáveis, levando ao edema da mucosa e redução de suprimento sanguíneo. Estes eventos determinam o aparecimento de um quadro clínico evolutivo que apresenta quatro fases: esbranquiçamento da mucosa, eritema, pseudomembrana e ulceração(7).

Entre os fatores de riscos que determinam maior susceptibilidade das células da mucosa à radioterapia e quimioterapia, os mais importantes são: cigarro, tipo de tratamento, idade e doenças crônicas como diabetes ${ }^{(9)}$.

Pesquisa realizada em $1990^{(10)}$ com 41 pacientes fumantes que receberam radioterapia hiperfracionada para o tratamento de tumor de cabeça e pescoço mostrou que os pacientes que paravam de fumar antes do início do tratamento apresentavam menor reação. Os pacientes foram divididos em quatro grupos: o primeiro incluía pacientes que nunca fumaram; o segundo grupo, os que pararam de fumar antes do início do tratamento; o terceiro grupo, os que pararam de fumar durante o tratamento, porém logo depois reiniciaram; e o quarto grupo com pa- 
cientes que não pararam de fumar em nenhum momento do tratamento. Observou-se que os pacientes do grupo 1 e 2 apresentaram mucosite que durou 13 semanas comparado com 21 semanas dos grupos 3 e $4^{(10)}$.

Por outro lado, pesquisa realizada com 115 pacientes com câncer de cabeça e pescoço submetidos à radioterapia e quimioterapia, concomitantes com objetivo de observar a influência do cigarro na resposta do tumor ao tratamento e na sobrevida, não demonstrou diferença entre o grupo de fumantes e não-fumantes em relação à severidade da mucosite, porém os pacientes que fumaram durante o tratamento tiveram uma queda na sobrevida(11).

Especificamente em relação à radioterapia, alguns fatores podem influenciar na severidade da mucosite tais como a dose total, volume tratado, fracionamento e tempo de tratamento ${ }^{(12)}$. Um estudo ${ }^{(13)}$ confirma a alta incidência de mucosite em pacientes que recebem radioterapia, sendo de $97 \%$ para aqueles que recebem radioterapia em esquema de fracionamento convencional, $100 \%$ para os submetidos à radioterapia com fracionamentos alterados (fracionamento acelerado ou hiperfracionado) e de $89 \%$ para os que recebem radioterapia em fracionamento convencional e quimioterapia concomitantes. Este mesmo estudo ressalta que esquemas de radioterapia acelerada podem influenciar na gravidade da mucosite: $53 \%$ dos pacientes que apresentaram mucosite graus 3 e 4 foram submetidos à radioterapia com alteração no fracionamento comparados com $34 \%$ dos que receberam fracionamento convencional e $43 \%$ para aqueles tratados com radioterapia em fracionamento convencional e quimioterapia concomitantes ${ }^{(13)}$. A incidência de mucosite graus 3 e 4 foi de $47 \%$ nos pacientes tratados com fracionamento acelerado ou hiperfracionamento. Nos pacientes que receberam 70Gy com uma fração diária de 200 cGy durante sete semanas, a incidência foi de $25 \%^{(12-13)}$.

Em relação à idade, os pacientes jovens submetidos a rádio e quimioterapia concomitantes são mais propensos a desenvolver mucosite devido a alta atividade mitótica das células epiteliais. Por outro lado, os idosos seriam menos propensos a mucosite pela baixa atividade mitótica. Porém, em relação aos pacientes idosos submetidos à quimioterapia, o declínio fisiológico da função renal pode contribuir para o desenvolvimento de mucosite em idades mais avançadas se não houver um ajuste de dose de acordo com a capacidade da função renal do paciente, pois com a diminuição da função renal aumenta o tempo para excreção da droga, fazendo com que ela permaneça no corpo por mais tempo e assim podendo causar mais danos. A relação entre a taxa de replicação celular e a severidade do dano causada pela radiação ionizante sugere que quanto maior a idade menor a severidade das reações ${ }^{(14)}$. No entanto, um outro estudo ${ }^{(15)}$ sugeriu que o reparo das células da mucosa oral é prejudicado com o envelhecimento, contribuindo para o desenvolvimento de uma mucosite mais severa.
Quanto ao diabetes, por apresentar repercussão sistêmica, acredita-se que esta doença possa influenciar a gravidade de mucosite. Trabalhos mostram que pacientes portadores de diabetes tipo I e II têm maior risco de desenvolverem gengivite e doença periodontal, devido à cicatrização prejudicada e à rápida degradação do colágeno pelas enzimas metaloproteinases ${ }^{(16)}$.

\section{MÉTODO}

Trata-se de um estudo observacional, tipo coorte, realizado no período entre janeiro a dezembro de 2006 em um hospital de ensino localizado na cidade de São Paulo e foi aprovado pelo Comitê de Ética e Pesquisa do hospital em questão sob o processo 0365/05.

Foram encaminhados para o Setor de Radioterapia 175 pacientes portadores de câncer de cabeça e pescoço, dos quais 50 pacientes preencheram os critérios de inclusão. Destes, 21 foram submetidos à cirurgia, seguido de radioterapia e quimioterapia adjuvante, e 29 receberam apenas radioterapia e quimioterapia concomitante.

Os critérios de inclusão do presente estudo foram: pacientes com mais de 18 anos de idade, com diagnóstico de câncer de cabeça e pescoço comprovado histologicamente nos seguintes sítios primários: cavidade oral, orofaringe, hipofaringe, laringe e seio maxilar, submetidos à radioterapia e quimioterapia concomitante e assinatura do termo de consentimento livre esclarecido.

Os critérios de exclusão adotados foram os seguintes: pacientes que apresentassem trismo acentuado impossibilitando realização a inspeção adequada da cavidade oral e orofaringe, pacientes submetidos a outro protocolo de tratamento quimioterápico, pacientes submetidos a radioterapia e/ou quimioterapia previamente e pacientes que não concluíram o tratamento inicialmente proposto.

Os pacientes foram submetidos à radioterapia com campos cérvico - faciais paralelos opostos, com dose total entre 66 a 70 Gy, em frações de 2gy/dia em aparelho de megavoltagem. Receberam quimioterapia com cisplatina na dose 30 $\mathrm{mg} / \mathrm{m} 2$ por semana ou carboplatina semanal calculado de acordo com o curva de concentração do plasma.

Os pacientes foram entrevistados pela enfermeira no dia do planejamento do tratamento. Nesta ocasião foi realizada a primeira avaliação da mucosa e, a partir de então, semanalmente durante todo o tratamento. Foram avaliados quanto ao grau de mucosite pelo radioterapêuta com acompanhamento da enfermeira.

Para a entrevista, foi preparado formulário contendo informações gerais, história com aspectos individuais de cada paciente e características da doença e do tratamento.

O critério adotado para avaliar o grau de mucosite foi o Common Toxicity Criterio - CTC, que é uma escala 
ordinal que apresenta quatro graus: Grau 0 - nenhuma mudança; Grau 1 - eritema da mucosa; Grau 2 - reação pseudomembranosa focal (placas $=1,5 \mathrm{~cm}$ de diâmetro e não contíguas); Grau 3 - reação psedomembranosa confluente (placas contíguas geralmente $>1,5 \mathrm{~cm}$ de diâmetro); Grau 4 - necrose ou ulceração profunda, podendo incluir sangramento não-induzido por trauma menor ou abrasão( ${ }^{(8)}$. Conforme a evolução da mucosite oral durante o tratamento foram tomadas medidas terapêuticas, dependendo do grau apresentado.

Os parâmetros referentes às características clínicas individuais, da doença, da mucosite (incidência global dos diferentes graus, incidência por região anatômica, graus de acordo com as semanas de tratamento) e as principais queixas relatadas pelos pacientes foram submetidas à análise descritiva.

Os parâmetros referentes à reação de mucosa, conforme as características clínicas individuais, foram submetidas à análise inferencial. Para análise inferencial, os pacientes foram agrupados da seguinte maneira: pacientes que não tiveram o tratamento suspenso por causa da mucosite (graus 0,1 e $2^{*}$ ) e pacientes que tiveram o tratamento suspenso por causa da mucosite (graus $2^{* *} \mathrm{e}$ 3). O critério adotado para suspensão do tratamento dos pacientes que apresentaram grau 2 foi o relato de disfagia e odinofagia, com comprometimento da alimentação e a presença de reação pseudomembranosa não-confluente porém multifocal.

Os testes estatísticos utilizados foram: teste exato de Fisher (P) e quiquadrado (x2).

Em todos os testes fixou-se em 0,05 ou 5\% (p<0,05) o nível de rejeição da hipótese de nulidade, assinalando-se com um asterisco os valores significantes e pela indicação (NS) os não significantes.

\section{RESULTADOS}

Neste estudo, 45 (90\%) dos pacientes foram do sexo masculino e 5 (10\%) do sexo feminino. A média de idade foi de 58 anos, 39 (78\%) dos pacientes pertenciam à etnia branca e 11 (22\%) à etnia negra. 40 (80\%) dos pacientes referiram ter parado de fumar antes do início do tratamento, enquanto oito (16\%) admitiram não ter parado de fumar e apenas dois (4\%) afirmaram nunca ter fumado. Do total de pacientes, 15 (30\%) eram hipertensos e apenas três (6\%) diabéticos.

Em relação ao sítio primário, 10 pacientes eram portadores de câncer de cavidade oral, 32 de orofaringe, quatro de hipofaringe, três de laringe e um de seio maxilar.

Observou-se predomínio de mucosite graus 1 e 2 (68\%) (Figura 1), com maior incidência na região da orofaringe (51\%) (Figura 2), entre as $3^{\text {a }}$ e $6^{\text {a }}$ semanas de tratamento (Figura 3).
Do total de pacientes, $86 \%$ tiveram o tratamento interrompido em algum momento, sendo que em $36 \%$ foi devido à mucosite, sendo o tempo médio de parada de 5,9 dias.

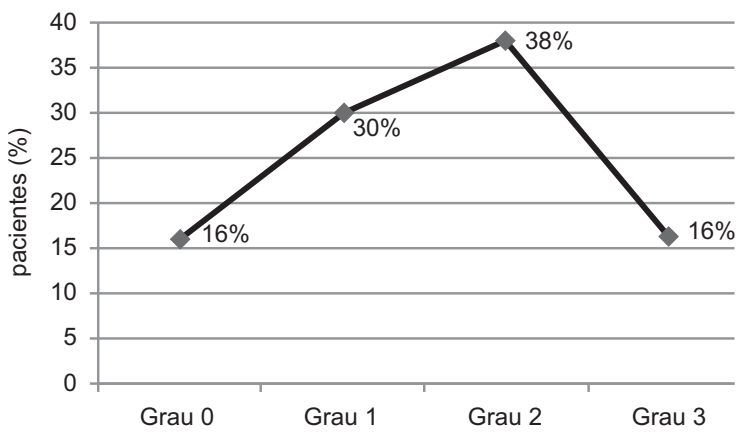

Figura 1 - Incidência dos diferentes graus de mucosite

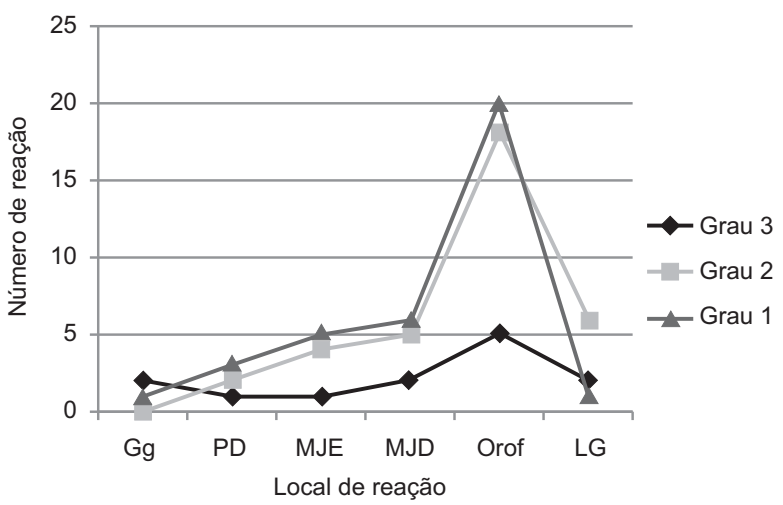

Legenda: Lg - língua; Orof - orofaringe; MJD - mucosa jugal direita $\mathrm{MJE}$ - mucosa jugal esquerda; PD - palato duro; Gg - gengiva.

Figura 2 - Incidência de mucosite conforme topografia

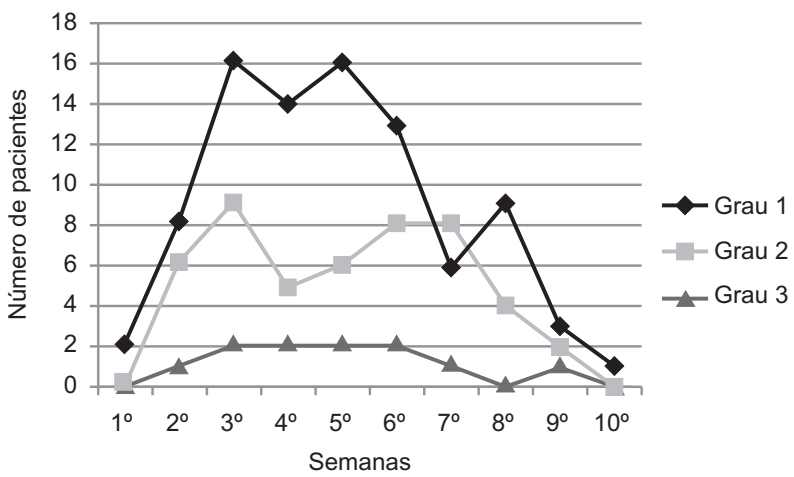

Figura 3 - Grau de mucosite de acordo com as semanas de tratamento

As principais queixas relatadas pelos pacientes após o início do tratamento foram perda do paladar (41\%) e xerostomia (29\%) (Figura 4). 


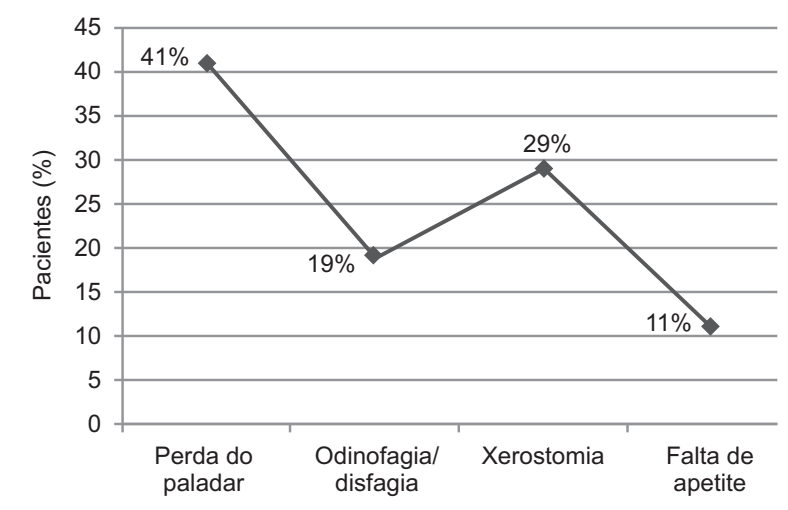

Figura 4 - Principais queixas relatadas pelos pacientes

A análise das características clínicas individuais dos pacientes, da doença e do tratamento mostrou que o diabetes aumenta significantemente a gravidade da mucosite (Tabela 1).

Tabela 1 - Tratamento interrompido (TI) e não interrompido (TNI) devido à mucosite classificada segundo o CTC 2.0 - São Paulo - 2005/2006

\begin{tabular}{|c|c|c|c|c|}
\hline & $\begin{array}{c}\text { TNI } \\
\text { Grau 0,1 e 2* } \\
\text { N (\%) }\end{array}$ & $\begin{array}{c}\text { TI } \\
\text { Grau 2** e } 3 \\
\text { N (\%) }\end{array}$ & $\begin{array}{c}\text { Total } \\
\text { N (\%) }\end{array}$ & $\mathbf{P}$ \\
\hline \multicolumn{5}{|l|}{ Faixa etária } \\
\hline Até 60 anos & $21(67,7)$ & $10(32,2)$ & $31(100)$ & \multirow[t]{2}{*}{0,96 (NS } \\
\hline Mais de 60 anos & $13(68,4)$ & $6(31,5)$ & $19(100)$ & \\
\hline \multicolumn{5}{|l|}{ Sexo } \\
\hline Feminino & $3(60)$ & $2(40)$ & $5(100)$ & \multirow[t]{2}{*}{$0,60(\mathrm{NS})$} \\
\hline Masculino & $31(68,9)$ & $14(31,1)$ & $45(100)$ & \\
\hline \multicolumn{5}{|l|}{ Etnia } \\
\hline Negra & $7(63,6)$ & $4(36,3)$ & $11(100)$ & \multirow[t]{2}{*}{0,49 (NS } \\
\hline Branca & $27(69,2)$ & $12(30,8)$ & $39(100)$ & \\
\hline \multicolumn{5}{|l|}{ Hipertensão } \\
\hline Sim & $10(66,7)$ & $5(33,3)$ & $15(100)$ & \multirow{2}{*}{$0,57(\mathrm{NS}$} \\
\hline Não & $24(68,6)$ & $11(31,4)$ & $35(100 \%)$ & \\
\hline \multicolumn{5}{|l|}{ Diabetes } \\
\hline Sim & 0 & $3(100)$ & $3(100)$ & \multirow[t]{2}{*}{$0,02^{*}$} \\
\hline Não & $34(72,3)$ & $13(27,7)$ & $47(100)$ & \\
\hline \multicolumn{5}{|l|}{ Fumo } \\
\hline Tabagista & $7(77,8)$ & $2(22,2)$ & $9(100)$ & \multirow[t]{3}{*}{$0,44(\mathrm{NS}$} \\
\hline Ex-tabagista & $25(64,1)$ & $14(35,9)$ & $39(100)$ & \\
\hline Não tabagista & $2(100)$ & 0 & $2(100)$ & \\
\hline \multicolumn{5}{|l|}{ Estadiamento } \\
\hline II & $4(100)$ & 0 & $4(100)$ & \multirow[t]{4}{*}{0,37 (NS } \\
\hline III & $6(60)$ & $4(40)$ & $10(100)$ & \\
\hline IVA & $19(63,3)$ & $11(36,7)$ & $30(100)$ & \\
\hline IVB & $5(83,3)$ & $1(16,7)$ & $6(100)$ & \\
\hline \multicolumn{5}{|l|}{ Cirurgia } \\
\hline Sim & $19(73,1)$ & $7(26,9)$ & $26(100)$ & \multirow[t]{2}{*}{0,54 (NS } \\
\hline Não & $15(62,5)$ & $9(37,5)$ & $24(100)$ & \\
\hline
\end{tabular}

Grau 2* tratamento não interrompido (TNI);

Grau 2 ** tratamento interrompido (TI).

\section{DISCUSSÃO}

A mucosite é uma complicação aguda bastante comum nos pacientes portadores de câncer de cabeça e pescoço que são tratados com quimioterapia e radioterapia.
Está associada ao desconforto e à dificuldade de ingestão e deglutição, podendo levar desde a interrupção do tratamento até ao uso de sonda nasoenteral e hospitalização ${ }^{(9)}$.

Observou-se no presente trabalho predomínio de mucosite graus $1(30 \%)$ e $2(38 \%)$, sendo o principal sítio encontrado a orofaringe. Isto se deve ao fato da maioria dos pacientes da amostra apresentarem tumor em orofaringe e cavidade oral. Na literatura, encontramos estudo com pacientes portadores de câncer de cabeça e pescoço que foram submetidos a diferentes tipos de tratamento: radioterapia e quimioterapia concomitantes, radioterapia com fracionamento convencional ou hiperfracionado, ou somente quimioterapia. Os resultados apresentados foram bem próximos aos encontrados neste estudo, no qual $83 \%$ dos pacientes apresentaram algum grau de mucosite; sendo que o grau moderado predominou em $35 \%{ }^{(17)}$. No entanto, em outro estudo, com pacientes portadores de câncer de cabeça e pescoço submetidos a diferentes modalidades de tratamento, $91 \%$ dos pacientes desenvolveram algum grau de mucosite, porém com predomínio do grau $3(60 \%)^{(18)}$.

Verificou-se maior incidência de mucosite entre a 3 a e 6a semanas de tratamento com predomínio das reações grau 1 e 2 . Outro estudo mostra maior incidência de mucosite entre a 3 a e 7a semana de tratamento com predomínio do grau 3, no qual os pacientes foram tratados com radioterapia e quimioterapia concomitantes, porém com alteração de fracionamento; o que poderia justificar a incidência maior de grau $3^{(18)}$. Outro fator é que em nosso estudo alguns pacientes tiveram o tratamento interrompido com grau 2 de mucosite, não permitindo a evolução da toxicidade. Na literatura encontramos estudos que afirmam que, com uma ou duas semanas de radioterapia, já é possível verificar eritema oral. Após a 2 a semana, com cerca de $20 \mathrm{~Gy}$, pode-se verificar a presença de edema; o eritema já não é tão visível devido à pressão exercida sobre os capilares; esta provocada pelo acúmulo de líquido extravascular. Depois de 3 semanas, com cerca de 30Gy, ocorre aumento da permeabilidade vascular, contribuindo para aumento do edema ${ }^{(19-20)}$. Baseado nestes trabalhos, pode-se perceber que os sinais começam a ficar mais evidentes a partir da 3 a semana; não se deixando de levar em conta a variabilidade individual de resposta.

Em relação aos fatores clínicos individuais, nossos resultados mostraram que a idade não foi fator importante para a gravidade da mucosite. Alguns autores referem que a chance de apresentar mucosite diminui com a idade. Isto seria devido à baixa taxa de replicação celular que ocorre no paciente idoso, uma vez que células com alta atividade mitótica são mais sensíveis à radioterapia e quimioterapia. Vale ressaltar que os pacientes que participaram deste estudo apresentaram média de idade menor (57,52 anos) quando comparados com a literatura (61,3 e 60,7 anos) ${ }^{(9,17)}$.

Com relação ao fumo, este fator não mostrou significância estatística, provavelmente devido à baixa incidên- 
cia de fumantes em nossa amostra. Os resultados mostraram que (16\%) admitiram fumar durante o tratamento e apenas dois tiveram o tratamento interrompido por causa da mucosite. Na literatura se encontra trabalhos que divergem em seus resultados; enquanto alguns não observaram influência do fumo na mucosite, outros afirmaram que o fumo teve influência quando o volume irradiado era menor $^{(10-11)}$. É importante considerar o fato de que os pacientes, nesses trabalhos, foram submetidos a tratamentos diferentes.

Com relação ao diabetes, apesar do pequeno número de pacientes da nossa amostra (6\%) apresentarem a doença, $100 \%$ tiveram o tratamento interrompido devido a gravidade da mucosite, fato evidenciado pela significância estatística. Na literatura, encontramos um trabalho que observou que pacientes portadores de câncer de cavidade oral e orofaringe, diabéticos e submetidos à radioterapia com fracionamento alterado apresentaram maior risco de desenvolver mucosite graus 3 e $4^{(17)}$. Observou-se, ainda, trabalhos nos quais os autores afirmam que o diabetes, por se tratar de uma doença sistêmica, pode influenciar na gravidade da mucosite e que esta patologia deve ser levada em consideração em pacientes irradiados na região da cabeça e pescoço(21).

De acordo com a literatura, pacientes portadores de diabetes tipo 1 e 2 têm maior risco de desenvolver gengivite e doença periodontal. Isto ocorre devido cicatrização prejudicada consequente à rápida degradação do colágeno pelas enzimas metaloproteinases. Além disto, o

\section{REFERÊNCIAS}

1. Parkin DM, Bray F, Ferlay J, Pisani P. Estimating the world cancer burden: Globocan 2000. Int J Cancer. 2001;94(2):153-6.

2. Brasil. Ministério da Saúde; Instituto Nacional do Câncer (INCA). Incidência de câncer no Brasil: estimativa 2010 [Internet]. Rio de Janeiro: INCA; 2009. [citado 2009 set. 15]. Disponível em: http://www.inca.gov.br/estimativa/2010/estimativa20091201.pdf

3. Rose-Ped AM, Bellm LA, Epstein JB, Trotti A, Gwede C, Fuchs $\mathrm{HJ}$. Complications of radiation therapy for head and neck cancers: the patient's perspective. Cancer Nurs. 2002;25(6):4617; quiz 468-9.

4. Denham JW, Peters LJ, Johansen J, Poulsen M, Lamb DS, Hindley $A$, et al. Do acute mucosal reactions lead to consequential late reactions in patients with head and neck câncer? Radiother Oncol. 1999;52(2):157-64.

5. Sonis ST, Elting LS, Keefe D, Peterson DE, Schubert M, Hauer-Jensen $M$, et al., Perspectives on cancer therapy-induced mucosal injury: pathogenesis, measurement, epidemiology, and consequences for patients. Cancer. 2004;100(9 Suppl):1995-2025. processo inflamatório é acelerado, o que dificulta a cicatrização, provoca potencialização da resposta de monócitos, induz altos níveis de proteínas glicosiladas, aumenta os produtos finais da glicação avançada (AGEs) nos tecidos e diminui a quimiotática dos neutrófilos ${ }^{(16,21-22)}$. Assim, baseados nas informações acima e em nossos resultados, acreditamos que pacientes diabéticos submetidos à radioterapia e quimioterapia, tratamentos que induzem inflamação, possam apresentar predisposição para o desenvolvimento de mucosite de maior gravidade uma vez que já possuem fatores que sensibilizam a mucosa oral.

\section{CONCLU5SÃO}

Analisados em conjunto, os dados do presente trabalho mostram que pacientes portadores de câncer de cabeça e pescoço submetidos à radioterapia e quimioterapia concomitante apresentam 13 predominâncias de mucosite graus 1 e 2 entre a 3 a e a 6 a semana de tratamento. Entre as características individuais dos pacientes, da doença e do tratamento, apenas o diabetes favoreceu o desenvolvimento de mucosite grave. Desta forma, acreditamos que pacientes diabéticos podem se beneficiar com acompanhamento mais amiúde durante o tratamento, com orientação e cuidados específicos, tais como controle da glicemia e da medicação. É importante que o enfermeiro esteja atento a estes parâmetros, pois, como profissional presente nos setores de radioterapia e quimioterapia, pode colaborar para otimização do tratamento e melhora da qualidade de vida dos pacientes.

6. Sawada NO, Nicolussi AC, Okino L, Cardozo FMC, Zago MMF. Quality of life evaluation in cancer patients to submitted to chemotherapy. Rev Esc Enferm USP [Internet]. 2009 [cited 2009 Sept 15];43(3):581-7. Available from: http://www.scielo. $\mathrm{br} / \mathrm{pdf} /$ reeusp/v43n3/en_a12v43n3.pdf

7. Naidu MUR, Ramana GV, Rani PU, Mohan IK, Suman A, Roy P. Chemotherapy-induced and/or therapy-induced oral mucositis-complicating the treatment of cancer. Neoplasia. 2004;6(5):423-31.

8. Saad ED, Hoff PM, Carnelós RP, Katz A, Novis YAS, Pietrocola $\mathrm{M}$, et al. Critérios comuns de toxicidade do Instituto $\mathrm{Na}$ cional de Câncer dos Estados Unidos. Rev Bras Cancerol. 2002;48(10):63-96.

9. Porock D. Factors influencing the severity of radiation skin and oral mucosal reactions: development of a conceptual framework. Eur J Cancer Care. 2002;11(1):33-43.

10. Rugg T, Saunders MI, Dische S. Smoking and mucosal reactions to radiotherapy. Br J Radiol. 1990;63(751):554-6. 
11. Browman GP, Wong G, Hodson I, Sathya J, Russeli R, Mcalpine $L$, et al., Influence of cigarette smoking on the efficacy of radiation therapy in head and neck cancer. N Engl J Med. 1993;328(3):159-63.

12. Mantini G, Manfrida S, Francesco C, Giammarino D, Petrone A, Vitucci $P$, et al. Impacto of dose and volume on radiation-induced mucositis. Rays. 2005;30(2):137-44.

13. Trotti A, Bellm LA, Epstein JB, Frame D, Fuchs HJ, Gwede CG, et al. Mucositis incidence, severity and associated outcomes in patients with head and neck cancer receiving radiotherapy with or without chemotherapy: a systematic literature review. Radiother Oncol. 2003;66(3):253-62.

14. Porock D, Nikoletti S, Cameron F. The relationship between factors that impair wound healing and the severity of acute radiation skin and mucosal toxicities in head and neck cancer. Cancer Nurs. 2004;27(1):71-8.

15. Gelman RS, Taylor SG. Cyclophosphamide, methotrexate, and 5-fluorouracil chemotherapy in women more than 65 years old with advanced breast cancer: the elimination of age trends in toxicity by using doses based on creatinine clearance. J Clin Oncol. 1984;2(12):1404-13.
16. Ryan ME, Carnu O, Kamer A. The influence of diabetes on the periodontal tissues. J Am Dent Assoc. 2003;134(Spec No):30S-40S.

17. Vera-Lionch $\mathrm{M}$, Oster $\mathrm{G}$, Hagiwara $\mathrm{M}$, Sonis S. Oral mucositis in patients undergoing radiation treatment for head and neck carcinoma. Cancer. 2006;106(2):329-36.

18. Elting LS, Cooksley CD, Chambers MS, Garden AS. Risk, outcomes, and costs of radiationinduced oral mucositis among patients with head-and-neck malignancies. Int J Radiat Oncol Biol Phys. 2007;68(4):1110-20.

19. Aziz L, Ebenfelt A. Mucosal secretion changes during radiotherapy in the oral cavity. Clin Oral Investig. 2007;11(3):293-6.

20. Baker DG. The radiobiological basis for tissue reactions in the oral cavity following therapeutic $x$-irradiation: a review. Arch Otolaryngol. 1982;108(1):21-4.

21. Barasch A, Peterson DE. Risk factors for ulcerative oral mucositis in cancer patients: unanswered questions. Oral Oncol. 2003;39(2):91-100.

22. Soell M, Hassan M, Miliauskaite A, Haikel Y, Selimovic D. The oral cavity of elderly patients in diabetes. Diabetes Metab. 2007;33 Suppl 1:S10-8. 